\title{
High Performance Modulation of High-Frequency AC Link Inverter
}

\author{
Abhijit Kulkarni and Vinod John \\ Department of Electrical Engineering, IISc Bangalore. \\ (abhijitk@ee.iisc.ernet.in, vjohn@ee.iisc.ernet.in)
}

\begin{abstract}
Inverters with high voltage conversion ratio are used in systems with sources such as batteries, photovoltaic (PV) modules or fuel cells. Transformers are often used in such inverters to provide the required voltage conversion ratio and isolation. In this paper, a compact high-frequency (HF) transformer interfaced AC link inverter with lossless snubber is discussed. A high performance synchronized modulation scheme is proposed for this inverter. This modulation addresses the issue of over-voltage spikes due to transformer leakage inductance and it is shown that the circuit can operate safely even when the turnon delay, such as dead-time, is not used in the HF rectifier section. The problem of spurious turn-on in the HF inverter switches is also mitigated by the proposed modulation method. The circuit performance is validated experimentally with a $900 \mathrm{~W}$ prototype inverter.
\end{abstract}

Index Terms-Photovoltaic systems, batteries, inverters, pulse width modulation.

\section{INTRODUCTION}

Inverters at a power level of the order of $1 \mathrm{~kW}$ using batteries, photovoltaic modules (PV) or fuel cells normally have low input voltage, typically less than $100 \mathrm{~V}$. High voltage conversion ratio is necessary to convert the input dc to line voltage ac which is typically $230 V_{r m s}, 50 H z$. To achieve the required voltage conversion ratio and to provide isolation, the inverter circuit topologies for such systems often use transformers [1]-[7].

High-frequency (HF) transformer interfaced AC link inverter is preferred in many applications due to compact size of the transformer. In this paper, HF AC link inverter topology shown in Fig. 1 is discussed. This topology has an HF inverter which converts the input dc to HF ac. The HF transformer steps up the voltage which is rectified by a high-frequency active rectifier. This topology needs a snubber to avoid the over-voltage spikes due to commutation with the transformer leakage inductance in the circuit [4]. Output of the rectifier contains a lossless snubber for efficiency improvement in place of the passive RC snubbers which are lossy [8]. The output stage consists of an H-bridge single-phase inverter. The output is shown to be grid connected in Fig. 1. It can also be used in stand-alone applications feeding local load.

The inverter topology must be modulated such that the overvoltage spikes are completely suppressed. This is achieved by the proposed high performance synchronized modulation

The authors would like to acknowledge the financial support from Department of Heavy Industry (DHI), Govt. of India, under the project "Offline and realtime simulator for Electric Vehicle/Hybrid Electric Vehicle systems". method. The proposed modulation results in ZVS turn-on of the HF inverter and rectifier switches. The snubber switch has ZVS turn-on and turn-off resulting in practically zero switching losses. This improves the overall system efficiency. The modulation also results in better dc voltage utilization compared to the quasi-square wave modulation. The quasisquare wave modulation intentionally introduces zero portions in the link voltage, hence reducing the average of the rectified AC link voltage.

Traditionally the switches of HF rectifier are provided with a turn-on delay, similar to the dead-time provided in an inverter [9]. It is shown in this paper, that such a turn-on delay along with the reverse recovery of the diodes can cause spurious turn-on of the devices in the HF inverter. This is an undesirable effect. The proposed modulation mitigates these spurious pulses, hence increasing the system reliability. The proposed modulation of the $\mathrm{HF}$ AC link inverter has been validated experimentally on a laboratory developed prototype.

This paper is organized as follows. Section II discusses the ideal operation of the inverter topology with the proposed modulation. The practical operation of the inverter and the problems of spurious turn-on of HF inverter devices due to the turn-on delay time (dead-time) is detailed in Section III. The effect of elimination of turn-on delay time in the HF rectifier to address the spurious turn-on problem is discussed in Section IV. The experimental results validating the proposed modulation on laboratory prototype are included in Section V. Conclusions are provided in Section VI.

\section{IdEAL MODES OF OPERATION OF THE HF TRANSFORMER INTERFACED INVERTER}

All the semiconductor devices in the inverter topology are assumed to be ideal. Thus the complementary devices are considered to be switched without any turn-on delay time. The proposed modulation for the ideal inverter includes the following synchronized switching.

1) The HF inverter and rectifier devices are switched in square wave mode in synchronism.

2) The output inverter is modulated using the conventional sine-triangle pulse width modulation (PWM) scheme.

3) The snubber device $S$ is turned on during the active state (AS) of the output inverter. That is, whenever the diagonal switches of the output inverter are ON, $S$ will be turned on. Thus, the switching frequency of $S$ will be twice that of output inverter. 


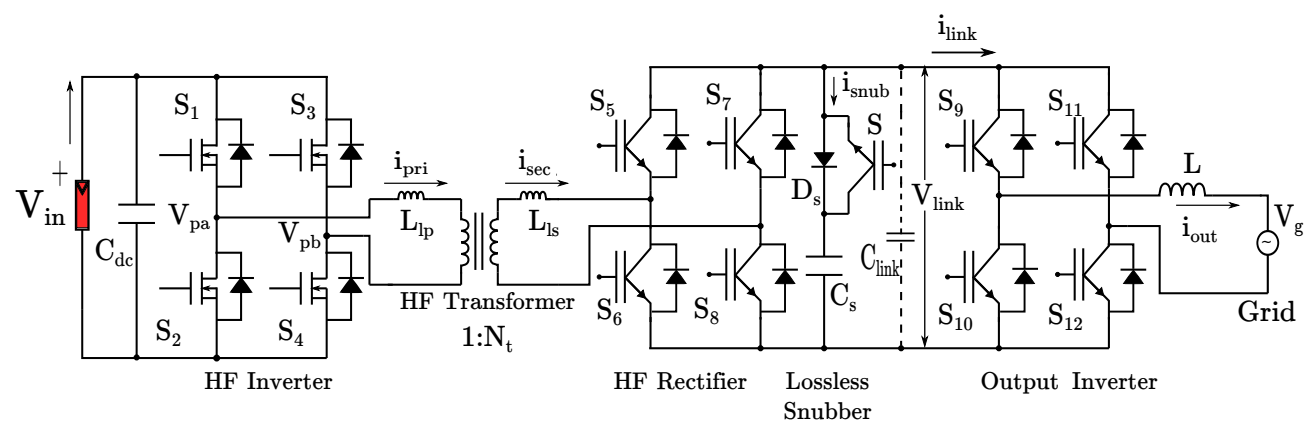

Fig. 1: HF transformer interfaced AC link inverter with a lossless snubber.

The modulation technique indicated in the three steps above, requires synchronization with appropriate phase shift of the carriers of HF inverter/rectifier and output inverter. The carriers used for the HF inverter, rectifier and output inverter are shown in Fig. 2(a) and (b). The time-relationship between output AS, zero-state (ZS) with link current, transformer primary voltage and current is shown in in Fig. 2(c) and (d).

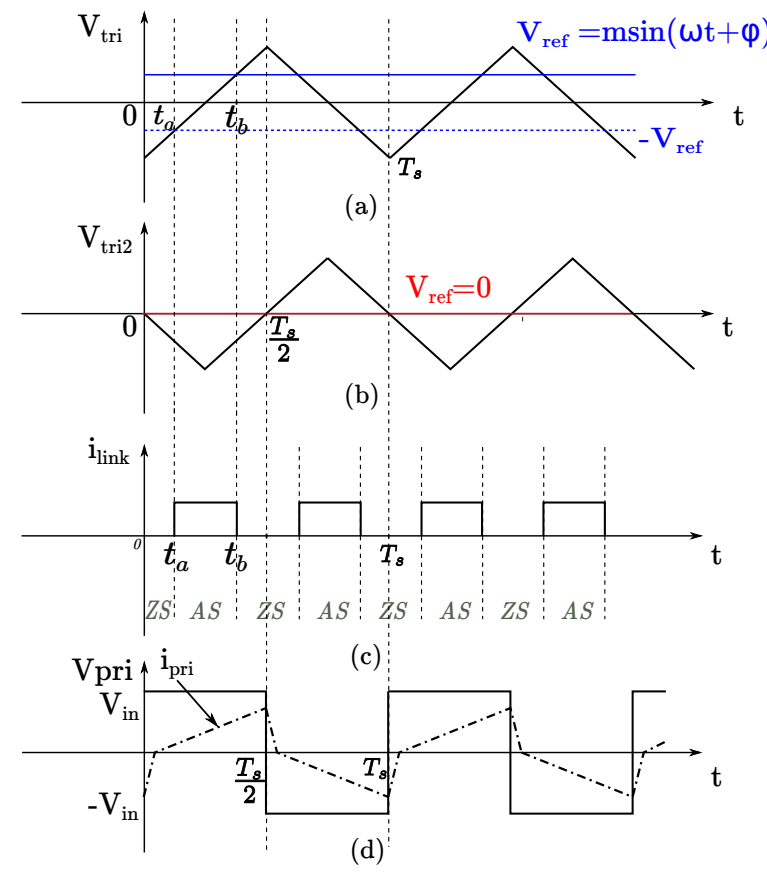

Fig. 2: (a) Triangular carrier for output inverter, (b) Triangular carrier for HF inverter and rectifier, (c) Link current indicating the active state and zero state and (d) Primary voltage and current.

The operation of the circuit, considering the different time intervals in a switching half-cycle, for the ideal condition is explained in the following subsections with reference to Fig. 2. The steps described in the following subsections will repeat in every switching half-cycle. The different modes of operation are described using equivalent circuit of the inverter topology. In the equivalent circuit, the devices $S_{9}, S_{10}, S_{11}, S_{12}$ and the output is replaced by a current source $i_{\text {link }}$. Each of the ideal modes of operation are detailed with an equivalent circuit which shows the conducting elements and gated-ON switches in dark lines. The non-conducting elements and the gated-OFF switches are shown in light-shades.

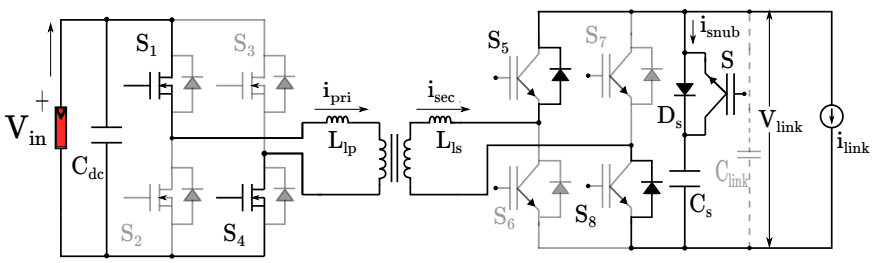

(a)

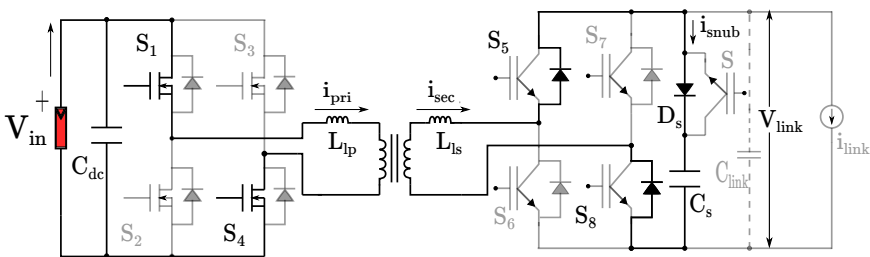

(b)

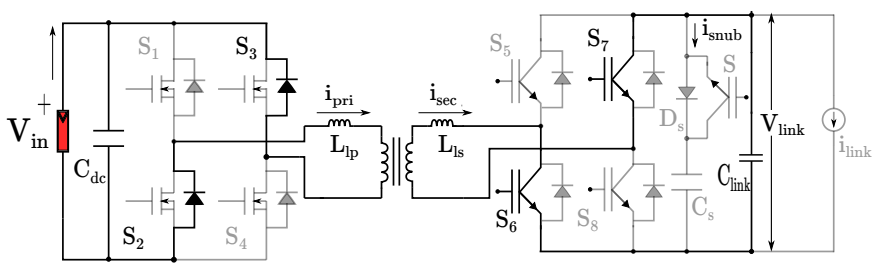

(c)

Fig. 3: Conducting devices for the ideal operation of the inverter in (a) Active state (AS), (b) Zero state (ZS), and (c) During primary voltage polarity reversal.

\section{A. For $t_{a} \leq t<t_{b}(A S)$}

In AS, magnitude of $i_{\text {link }}$ equals that of output current. $S$ is turned on and hence $C_{s}$ discharges through $S$ to supply the necessary $i_{l i n k}$. This prevents a large $d i / d t$ for transformer current. This means no voltage spike on the semiconductor devices. Primary and secondary currents $\left(i_{p r i}, i_{s e c}\right)$ are building up in positive direction. The equivalent circuit in AS is shown in Fig. 3(a), indicating various conducting and non-conducting paths.

\section{B. For $t_{b} \leq t<T_{s} / 2(Z S)$}

$i_{\text {out }}$ circulates between $S_{12}$ and diode of $S_{10}$ hence making $i_{\text {link }}=0 . V_{p r i}$ is positive and $C_{s}$ is being charged via $D_{s}$. 
Snubber switch $S$ is off. The equivalent circuit during this mode is shown in Fig. 3(b).

\section{For $t>T_{s}^{+} / 2$ upto next $A S$}

This interval includes the zerostate after the polarity reversal in the primary voltage, which occurs at $t=n T_{s}^{+} / 2$ where $n$ is any integer. $i_{\text {sec }}$ starts flowing through $S_{6}, C_{\text {stray }}$ and $S_{7}$ since $S_{5}, S_{8}$ are turned off, as shown in Fig. 3(c). The snubber switch and diode do not participate in the conduction of $i_{\text {sec }}$ as $S$ is off and $D_{s}$ cannot conduct reverse current. Hence, $i_{s e c}$ discharges $C_{\text {stray }}$ resetting the link voltage to zero. Secondary voltage $V_{\text {sec }}$ also goes to zero during this reset.

Let $I_{s e c}$ be the value of secondary current when the link voltage reset happens. As the energy in leakage inductance is significantly higher than the energy stored in stray capacitance $C_{\text {link }}$, there will be practically negligible change in the value of $I_{s e c}$. Thus the slope with which the link voltage gets reset is given by

$$
\frac{d V_{\text {link }}}{d t}=\frac{I_{\text {sec }}}{C_{\text {link }}}
$$

Then $i_{s e c}$ and $i_{p r i}$, with value $I_{s e c}$ and $n I_{s e c}$ respectively, will decay to zero with the following slope.

$$
\left|\frac{d i}{d t}\right|=\frac{V_{i n}}{L_{l k}}
$$

In (2), $L_{l k}$ is the effective leakage inductance referred to the primary side. The leakage inductances $L_{l p}$ and $L_{l s}$ in (3) below are indicated in Fig. 1. If $1: N_{t}$ be the turns ratio of the transformer, then

$$
L_{l k}=L_{l p}+\frac{L_{l s}}{N_{t}^{2}}
$$

Note that the link voltage cannot become negative due to the presence of anti-parallel diodes in $S_{5}, S_{6}, S_{7}$ and $S_{8}$. Thus, once reset to zero, link voltage stays at zero till the transformer current reaches zero. The resetting of link voltage and secondary voltage when the transformer primary voltage changes polarity is shown in Fig. 4. These waveforms are zoomed around the time instant when the polarity reversal of primary voltage happens. The gate to source voltages $\left(V_{G S}\right)$ of primary side switches $S_{1}, S_{2}$ and secondary side switches $S_{5}, S_{6}$ are also shown in Fig. 4. The drain to source voltage $\left(V_{D S}\right)$ of $S_{2}$ is also shown. The secondary voltage with its zero portion between $t_{1}$ and $t_{2}$ is shown. The polarity reversal in the primary voltage occurs at $t=t_{1}$ which is equal to $n T_{s} / s$ as stated before. It can be seen that the falling and rising slopes of $V_{s e c}$ are unequal. This is because, the falling of $V_{s e c}$ occurs at a higher value of $i_{s e c}$ (or $i_{p r i}$ ) while the rising occurs when the transformer currents are small. This is reflected in the link voltage also.

\section{Practical Operation of the HF Transformer INTERFACED INVERTER}

The proposed modulation is analysed for the practical implementation in this section. Normally, turn-on delay or dead-time is provided for all the complementary switching
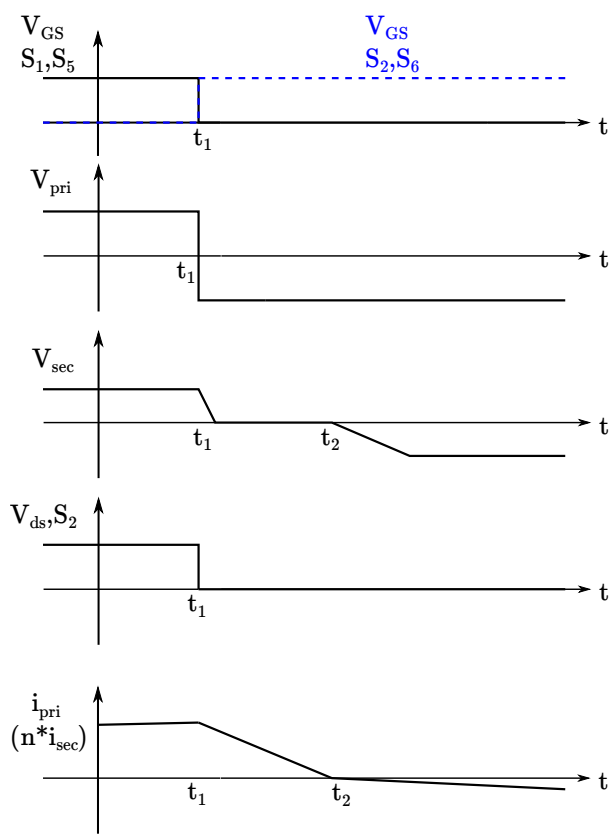

Fig. 4: Analytical waveforms for the ideal case zoomed around the instant of polarity reversal in the transformer primary voltage.

devices [9]. Fig. 5 shows the waveforms for the normal implementations with a turn-on delay time of $t_{d}$. This can be compared with the same set of waveforms in Fig. 4. The effect of diode reverse recovery is considered for the practical behaviour analysis. The various key waveforms are labelled in in Fig. 5. The occurrence of false turn-on in primary voltage and $V_{D S}$ of $S_{2}$ can be seen in the figure. This is explained in detail in the following subsections.

\section{A. For $0<t \leq t_{1}$}

Switches $S_{1}, S_{5}$ are on. $V_{p r i}, V_{\text {sec }}$ and $i_{p r i}, i_{s e c}$ are positive. $i_{\text {pri }}$ flows through $S_{1}$ and $S_{4}$ in the primary side and $i_{s e c}$ through the reverse diodes of $S_{5}$ and $S_{8}$ in the secondary side. This time interval corresponds to the zero state as just before the primary voltage polarity reversal, the output inverter is in zero state as can be seen from Fig. 2. Hence, the equivalent circuit for this time interval will be same as Fig. 3(b).

B. For $t_{1}<t \leq t_{2}^{\prime}$

This is an interval during dead-time. This circuit mode is absent in the ideal operation of the circuit. The equivalent circuit is shown in Fig. 6(a). $S_{1}, S_{5}$ and their paired devices $S_{4}, S_{8}$ are turned off. The $i_{p r i}$ starts to flow through the diodes of $S_{2}$ and $S_{3} \Rightarrow$ hence reversing the primary voltage polarity. Secondary voltage continues to be positive because the diodes of $S_{5}$ and $S_{8}$ were conducting and the turn-off of their corresponding IGBTs will not have any impact. Due to the application of a large negative voltage across leakage inductance, the $i_{p r i}$ and $i_{\text {sec }}$ start to fall linearly. The rate of fall is given by

$$
|d i / d t|=\frac{\left(V_{i n}+\left|V_{l i n k}\right| / N_{t}\right)}{L_{l k}}
$$




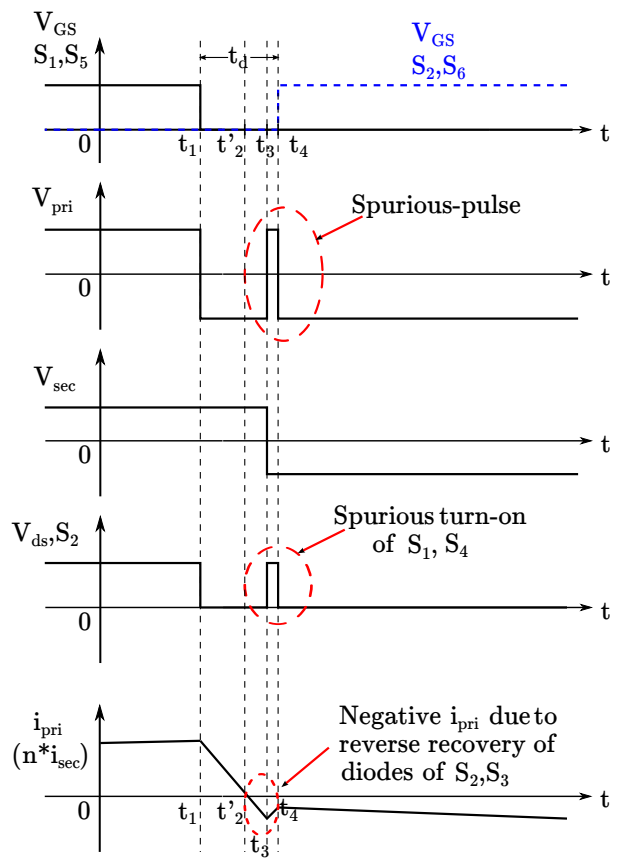

Fig. 5: Analytical waveforms zoomed around the instant of polarity reversal in the transformer primary voltage for the practical case with turn-on delay time $t_{d}$.

At time $t=t_{2}^{\prime}$, the $i_{p r i}$ and $i_{\text {sec }}$ go to zero.

C. For $t_{2}^{\prime}<t \leq t_{3}$

Ideally, the $i_{p r i}$ and $i_{s e c}$ should have remained clamped at zero. However, they go negative due to diode reverse recovery. At $t=t_{3}$, the diodes of $S_{2}, S_{3}$ and $S_{5}, S_{8}$ turnoff. The equivalent circuit for this case is same as Fig. 6(a) but with the polarities of $i_{p r i}$ and $i_{s e c}$ reversed. This is shown in Fig. 6(b) with the reversed current direction. Note that all the diodes, including the snubber diode $D_{s}$, are conducting negative current due to the diode reverse recovery.

\section{For $t_{3}<t \leq t_{4}$}

The $i_{\text {pri }}$ is now slightly negative but the dead-time duration is still persisting. As, $i_{\text {pri }}$ cannot be interrupted suddenly, it flows through the diodes of $S_{1}, S_{4}$ in the primary side. This gives rise to positive primary voltage spurious-pulse which is not expected from ideal waveforms. This spurious turn-on can be seen in the $V_{D S}$ of $S_{2}$ and $S_{3}$ also.

The secondary current will be carried by the diodes of $S_{6}$, $S_{7}$ resulting in $V_{s e c}$ becoming negative. Now the net positive voltage applied across leakage inductance, tries to increase the $i_{p r i}$ and $i_{s e c}$. The equivalent circuit for this mode is shown in Fig. 6(c). It can be observed that the net voltage applied across the leakage inductance is positive now.

\section{E. For $t>t_{4}$ till next $A S$}

The dead-time ends at $t=t_{4}$. Thus the $V_{p r i}$ changes its polarity to the actual expected value and the currents will have the ideally expected slope. This is shown in the equivalent circuit in Fig. 6(d). The transformer voltages and currents are now negative.

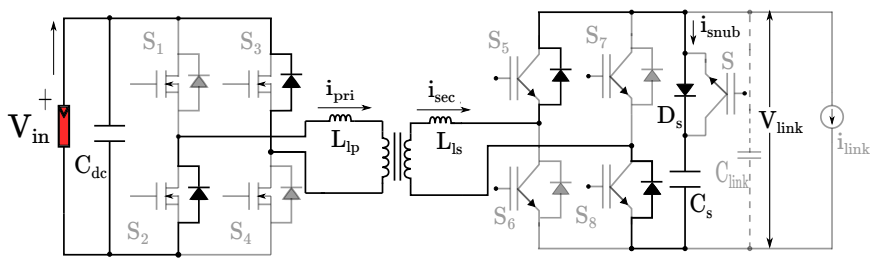

(a)

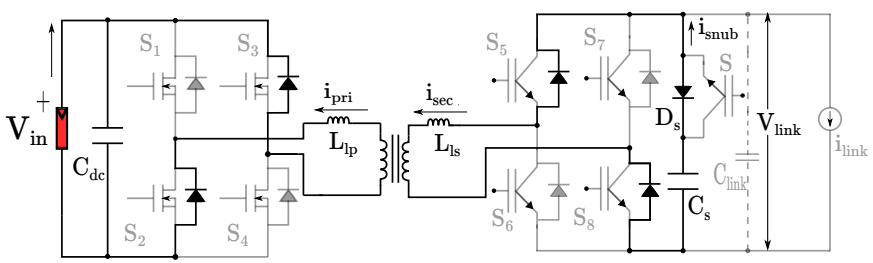

(b)

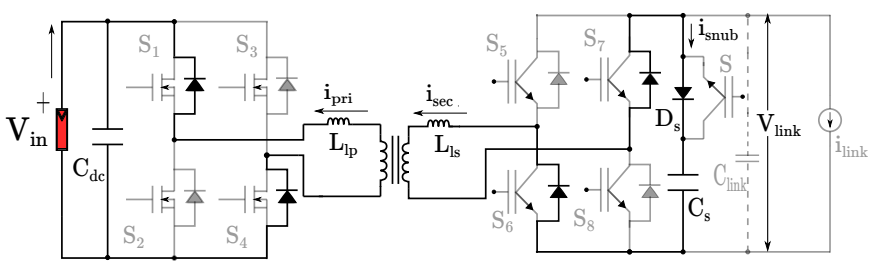

(c)

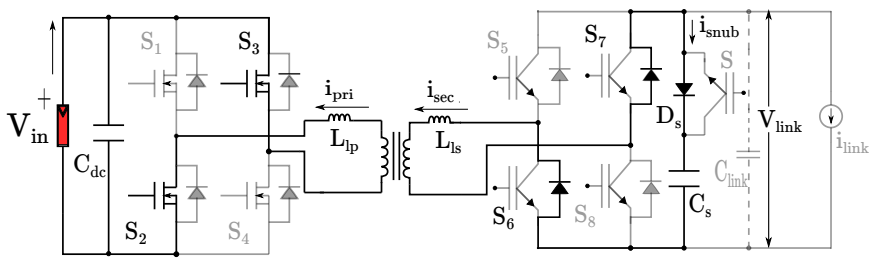

(d)

Fig. 6: Practical operation with dead-time. (a) During the deadtime with only diode conduction. (b) During dead-time with all the diodes conducting negative current due to reverse recovery. (c) During dead-time with spurious turn-on of diodes of $S_{1}$ and $S_{2}$. (d) End of the dead-time with output inverter still in zero state.

The practical operation of the circuit can be explained similarly when the polarity reversal occurs from negative to positive in the primary voltage.

\section{ELIMINATION OF SPURIOUS TURN-ON IN THE HF-INVERTER SWITCHES}

It was detailed in Section III that the turn-on delay time causes false turn-on pulses in the HF inverter switches. This problem can be solved if the turn-on delay time is eliminated in the $\mathrm{HF}$ rectifier switches namely $S_{5}, S_{6}, S_{7}$ and $S_{8}$. It is also shown that this can be safely done without any shoot through issues in the circuit. The operation of the circuit when the turn-on delay time in HF rectifier is eliminated is explained as follows. The time intervals detailed below are with respect to Fig. 7. 


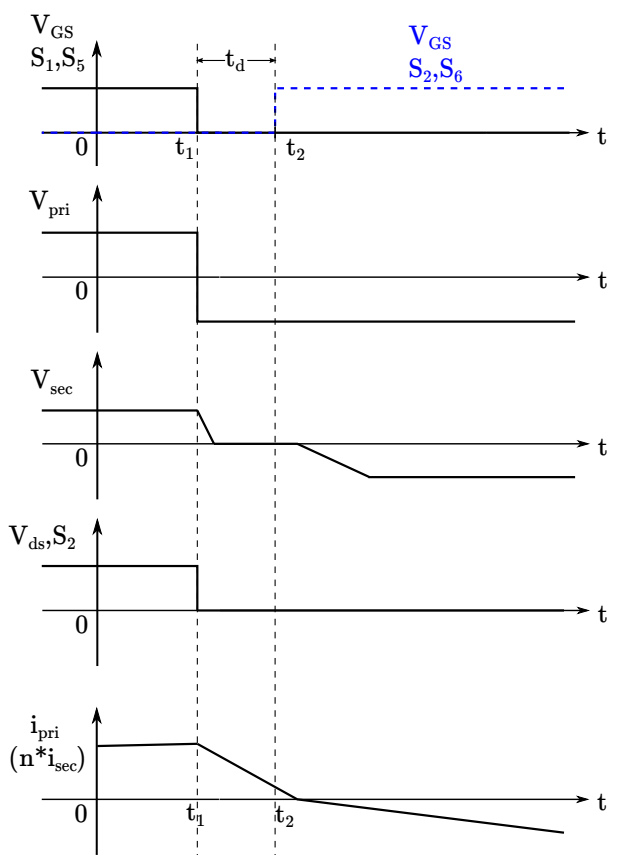

Fig. 7: Analytical waveforms zoomed around the instant of polarity reversal in the transformer primary voltage for the proposed modulation.

\section{A. For $0<t \leq t_{1}$}

This is same as that for practical case explained in Section III. The equivalent circuit for this time interval will be same as Fig. 3(b).

\section{B. For $t_{1}<t \leq t_{2}$}

This is an interval during dead-time in HF inverter. As in case of the operation in Section III, the primary side conduction is by the diodes of $S_{2}$ and $S_{3}$. The input voltage polarity reverses just at the beginning of dead-time, as expected in ideal case.

The HF rectifier switches do not have a dead-time. Thus, $S_{5}, S_{8}$ are turned off and their complementary devices $S_{6}$ and $S_{7}$ are turned-on simultaneously. There can be a switching overlap of the rectifier switches at this instant. The equivalent circuit during this time instant is shown in Fig. 8.

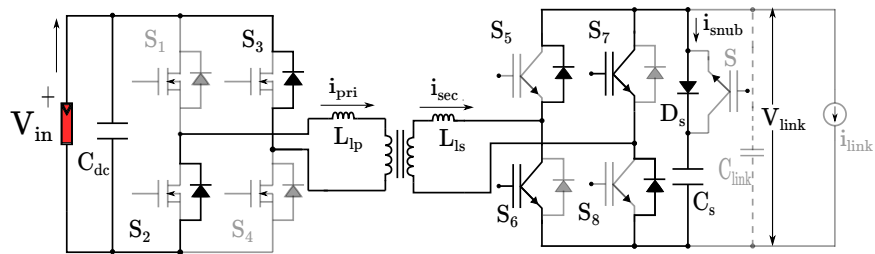

Fig. 8: The equivalent circuit with proposed modulation showing the commutation in the secondary side when the primary side switches have dead-time interval.

The diodes of $S_{5}$ and $S_{8}$ will turn-off completely during the dead-time period of the HF inverter, transferring the complete secondary current to $S_{6}$ and $S_{7}$. The resulting current will discharge the link voltage to zero as it does not have a reverseflow path due to $D_{s}$ and the snubber IGBT $S$ is OFF as the commutation occurs in the $\mathrm{ZS}$ of the output inverter. The equivalent circuit for this time instant will be identical to Fig. 3(c).

The shorting of link voltage and application of negative primary voltage causes the transformer current to drop. The transformer currents start falling at a slope given in (2) which is the ideally expected value. The dead-time ends at $t=t_{2}$.

\section{For $t>t_{2}$}

The HF inverter switches $S_{2}$ and $S_{3}$ are turned on while their diodes are conducting. Hence it is a ZVS turn-on. The transformer currents continue to fall with the slope given in (2) and reach zero. Then the polarity of currents changes to negative and the diodes of $S_{6}$ and $S_{7}$ in HF rectifier begin to conduct.

The secondary current charges up the link voltage till it exceeds to forward bias the snubber diode. Then the link voltage equals the snubber capacitor voltage and the system continues in this state till the next active state begins. The equivalent circuit for this will be same as Fig. 6(d).

\section{EXPERIMENTAL RESUlts}

When there is turn-on delay present in both HF inverter and rectifier devices, there will be false turn-on as explained in Section III. This is shown in Fig. 9(a).

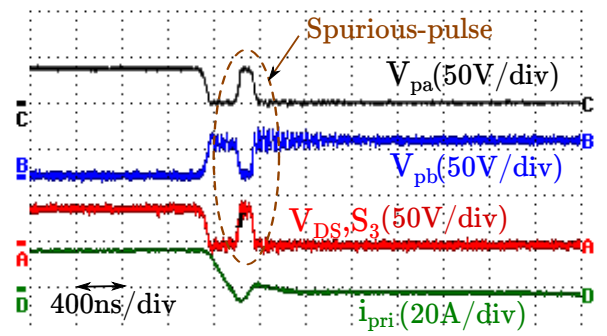

(a)

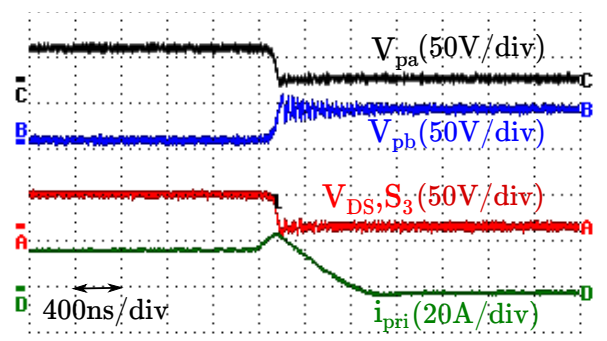

(b)

Fig. 9: (a) Spurious turn-on when turn-on delay is present in $\mathrm{HF}$ rectifier switches, (b) Improved performance with simultaneous switching in HF rectifier switches.

The voltages $V_{p a}, V_{p b}$ are marked in Fig. 1 and their difference is the $V_{p r i}$. The same set of waveforms are obtained with the proposed modulation in Fig. 9(b) . Clearly, there is no spurious turn-on pulse with proposed modulation. The primary current is also shown in Fig. 9(a) and (b). Due to the link voltage reset, the $d i_{p r i} / d t$ for the case with proposed modulation is lesser. This aids in the ZVS turn-on for the HF inverter switches. 


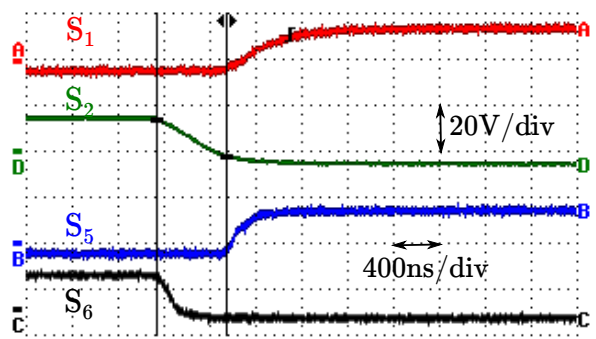

(a)

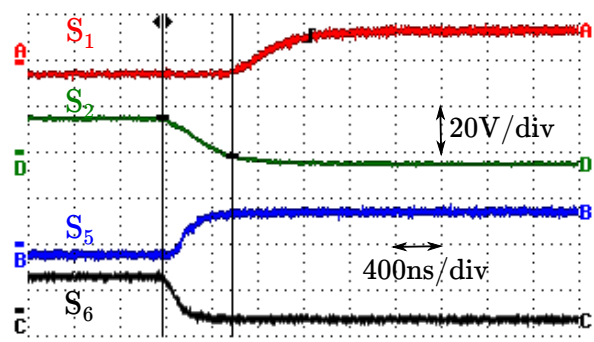

(b)

Fig. 10: $V_{G S}$ signals for $S_{1}, S_{2}, S_{5}$ and $S_{6}-$ (a) With conventional turn-on delay, (b) Proposed simultaneous switching for HF rectifier switches (Shown for $S_{5}$ and $S_{6}$ ).

The gate-to-source voltages for $S_{1}, S_{2}$ and $S_{5}, S_{6}$ are shown in Fig. 10(a) and (b). It can be observed that in Fig. 10(a) there is a turn-on delay between both pairs of complementary devices which is as per the conventional implementation. In Fig. 10(b), the proposed simultaneous switching for HF rectifier switches $S_{5}$ and $S_{6}$ is shown.

The secondary voltage and current at a frequency of $20 \mathrm{kHz}$ are shown with the proposed modulation in Fig. 11(a). In Fig. 11(b), the output voltage and currents are shown. The system has been tested in stand-alone mode for upto $0.9 \mathrm{~kW}$.

When the inverter was modulated with the traditional turnon delay time in $\mathrm{HF}$ rectifier switches, the circuit behaviour was very noisy due to spurious pulses. It could be run without trip-signal from protection circuitry only upto an input voltage of $30 \mathrm{~V}$ and a power level of $389 \mathrm{~W}$. The losses were determined to be $57.8 \mathrm{~W}$. At the same power level, with the proposed modulation, the losses were reduced to $52.4 \mathrm{~W}$.

\section{CONCLUSION}

In this paper, a high performance synchronized modulation is proposed for a $\mathrm{HF}$ AC link inverter with low input voltage for PV, battery or fuel cell input systems. This modulation addresses the over-voltage spikes in the circuit due to transformer leakage inductance. Practical operational issue of spurious turn-on is analyzed and solved by using the proposed simultaneous switching in the HF rectifier switches. It is shown that the turn-on delay time in HF rectifier section can be eliminated without causing any shoot-through problems. The HF inverter and rectifier switches can have soft switching with the proposed modulation. The performance of the circuit is validated experimentally with the proposed modulation.

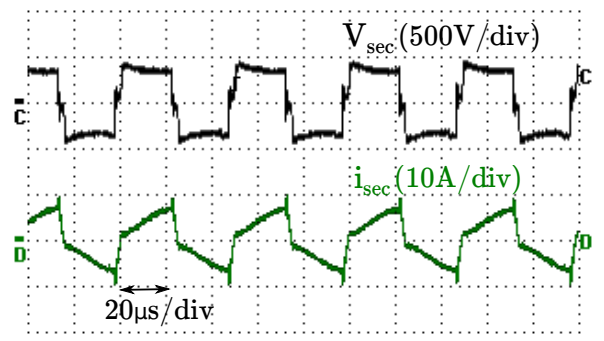

(a)

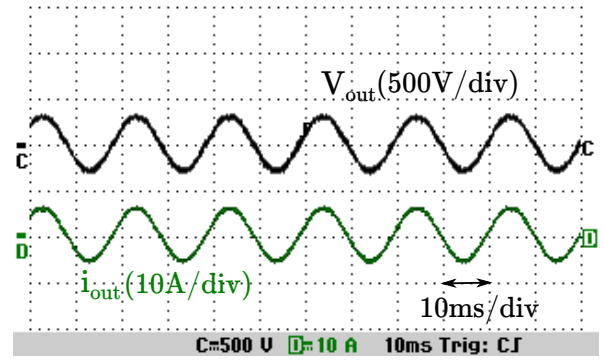

(b)

Fig. 11: (a) Transformer secondary voltage and current, (b) Output current and voltage at a power level of $0.9 \mathrm{~kW}$.

\section{REFERENCES}

[1] Z. Salam, S. Ayob, M. Ramli, and N. Azli, "An improved 'dc-dc type' high frequency transformer-link inverter by employing regenerative snubber circuit," in Power Electronics and Drive Systems, 2007. PEDS '07. 7th International Conference on, pp. 1081-1084, Nov 2007.

[2] W. Li, L. Fan, Y. Zhao, X. He, D. Xu, and B. Wu, "High-step-up and highefficiency fuel-cell power-generation system with active-clamp flybackforward converter,' IEEE Transactions on Industrial Electronics, vol. 59, pp. 599-610, Jan 2012.

[3] A. Kulkarni and V. John, "Mitigation of lower order harmonics in a grid-connected single-phase pv inverter," IEEE Transactions on Power Electronics, vol. 28, pp. 5024-5037, Nov 2013.

[4] T. Shimizu, K. Wada, and N. Nakamura, "Flyback-type single-phase utility interactive inverter with power pulsation decoupling on the dc input for an ac photovoltaic module system," Power Electronics, IEEE Transactions on, vol. 21, pp. 1264-1272, Sept 2006.

[5] D. Martins and R. Demonti, "Grid connected pv system using two energy processing stages," in Photovoltaic Specialists Conference, 2002. Conference Record of the Twenty-Ninth IEEE, pp. 1649-1652, May 2002.

[6] J. Mapurunga Caracas, G. De Carvalho Farias, L. Moreira Teixeira, and L. De Souza Ribeiro, "Implementation of a high-efficiency, high-lifetime, and low-cost converter for an autonomous photovoltaic water pumping system," IEEE Transactions on Industry Applications, vol. 50, pp. 631641, Jan 2014.

[7] B. Zhao, Q. Song, W. Liu, and Y. Sun, "Overview of dual-active-bridge isolated bidirectional $\mathrm{dc}-\mathrm{dc}$ converter for high-frequency-link powerconversion system," IEEE Transactions on Power Electronics, vol. 29, pp. 4091-4106, Aug 2014.

[8] D. De and V. Ramanarayanan, "A proportional + multiresonant controller for three-phase four-wire high-frequency link inverter," IEEE Transactions on Power Electronics, vol. 25, pp. 899-906, April 2010.

[9] J.-W. Choi and S.-K. Sul, "Inverter output voltage synthesis using novel dead time compensation," Power Electronics, IEEE Transactions on, vol. 11, pp. 221-227, Mar 1996. 